\title{
ON THE AUTOMATIC ELECTRONIC ASTROLABE
}

\author{
IETSUNE TSUBOKAWA \\ Earthquake Research Institute, The University of Tokyo, Tokyo, Japan \\ and \\ SHUNRO HOKUGO \\ Geographical Survey Institute, Ministry of Construction, Tokyo, Japan
}

\begin{abstract}
The pendulum astrolabe with an electronic transit detector after the principle proposed by I. Tsubokawa has comparable accuracy to those of the Danjon astrolabe and P.Z.T. even in the field. After the same principle, an instrument for observatory use is being constructed, in which all observational processes are done automatically under the predetermined programs. The necessary mechanisms for automatic operation are as follows:

(1) Azimuth setting mechanism for respective stars observed.

(2) Mechanism which turn the telescope through $180^{\circ}$ during the passage of a star near the mean altitude.

(3) Tracking mechanism in azimuth direction.

(4) Tracking mechanism in altitude direction.

(5) Read-out mechanism of the times of passages of a star on respective altitude circles.

Of these, the altitude tracking mechan ism directly affects the observational accuracy. The rotation of a plane parallel glass compensates the diurnal motion of a star and keeps its image in the state of repose on the edge of a prismatic knife of the electronic detector. The rotating mechanism gives to the plane glass combined rotation of the two parts; the servo-system of the electronic detector and the system of generating the variable rotational velocity corresponding to the diurnal motion of a star. The extreme accuracy in the earth's atmosphere is expected.
\end{abstract}

We are now developing an observatory type astrolabe. Although the general principle of the instrument is the same as that of the field type after I. Tsubokawa, it has further the following two special features:

(1) It excludes the effect of various kinds of heat sources. Among these the human observer, a source of more than a hundred watts is considered to be the largest. This necessitates the automatic operation of the instrument.

(2) In order to secure extreme accuracy, precise tracking mechanisms of a star image are used.

Figure 1 shows the general construction of the astrolabe after Tsubokawa. It consists of four parts: (1) A mirror system which deflects the ray from a star by a constant angle, that is $30^{\circ}$. (2) A telescope whose collimation line is maintained vertical. (3) The detector which determines the time of transit of a star image without personal equation. (4) The guiding telescope. The incident light from a star is reflected at the mirror $M_{1}$ and again reflected at the mirror $M_{2}$. If the angle of intersection of the two mirrors is $15^{\circ}$, then the ray from a star of which the altitude is $60^{\circ}$ becomes exactly vertical after the reflections regardless of a small inclination of the mirror system. The vertical ray passing through the first nodal point of the objective will be emitted from the second nodal point $K_{2}$ vertically downward, and then reflected at the freely suspended mirror surface $M$ which is situated horizontally at the distance of one half of the focal 
length of the objective. This reflected ray will again pass through the second nodal point in the same way as in P.Z.T., and thus the star image at the nodal point corresponds to the ray of a star whose zenith distance is twice the angle of intersection of the two mirrors in spite of a small inclination of the instrument. As it is not convenient to place the actual reference system in a plane through the nodal point $K_{2}$, the light from the freely suspended mirror $M$ is further reflected at a prism, which is rigidly connected with the objective. As is easily seen, the mirror image $R$ of the point $K_{2}$ is equivalent to $K_{2}$ in effect, and the line set here perpendicular to the paper corresponds to the

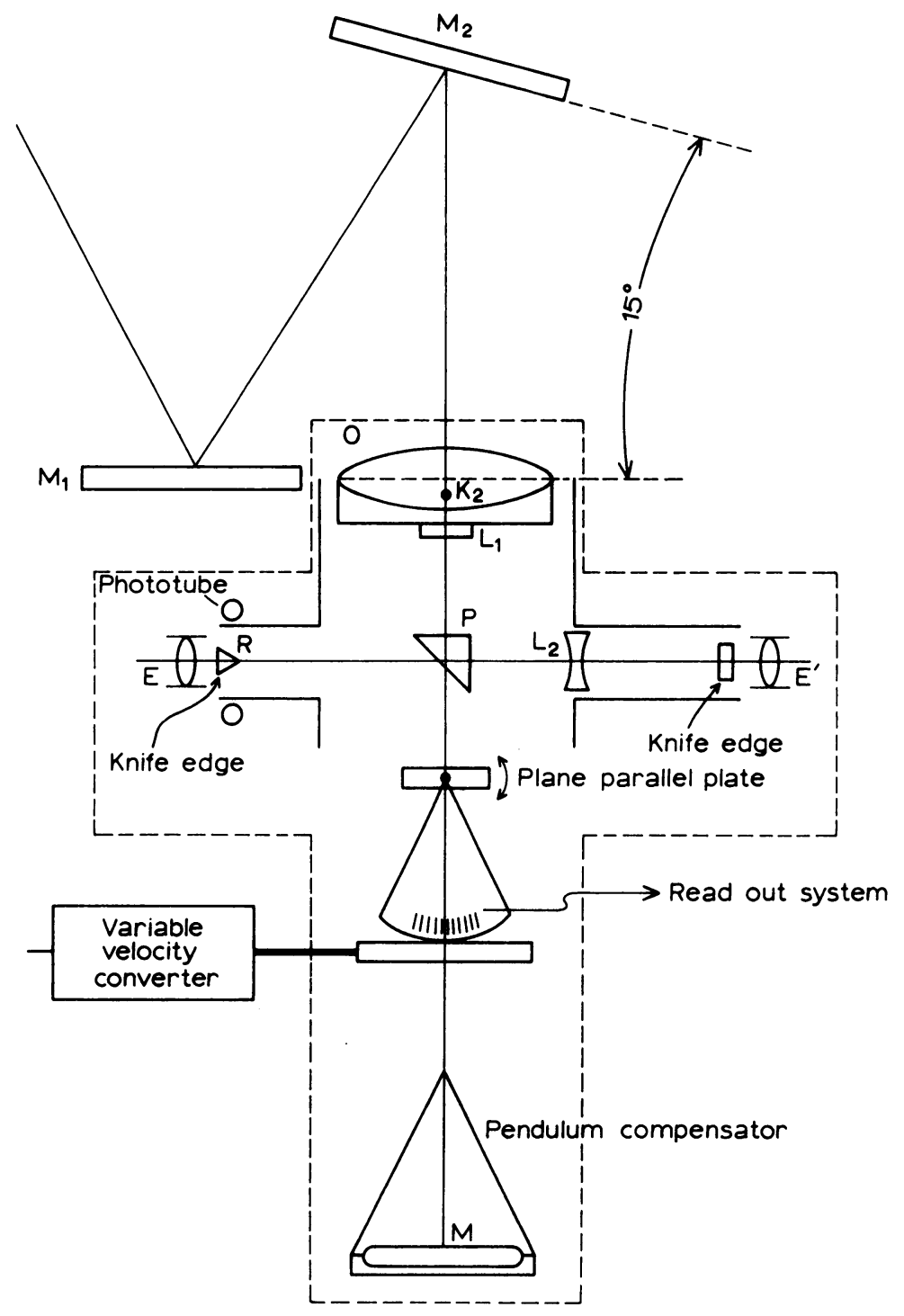

Fig. 1. General construction of the automatic electronic astrolable. 
altitude circle of $60^{\circ}$. If a group of time detecting knife-edges are set symmetrically to the central line through $R$, like the hairs in a transit instrument and the telescope which is enclosed by a dotted line in the figure is revolved by $180^{\circ}$ about the collimation line before the star image attains to the central line, we may eliminate various kinds of instrumental errors and determine the time of transit of a star referred to the central line. The time detector using these knife-edges is called E.T.D. (Tsubokawa, 1954a, b; Tsubokawa and Seto, 1957; Tsubokawa and Dambara, 1957).

The principle of this device is shown in Figure 2. $O$ is the objective and $N$ is the prismatic shaped knife of which the edge is placed on the focal plane of the objective.

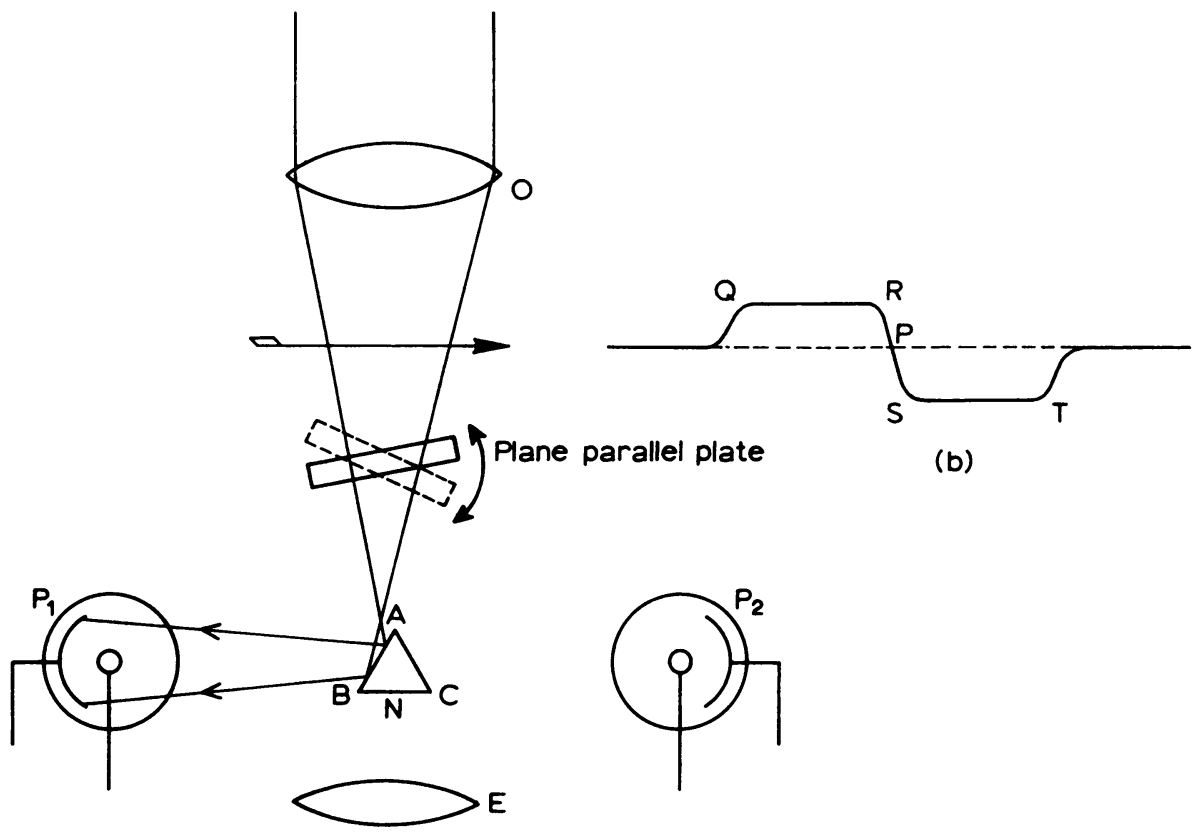

(a)

Fig. 2. The principle of E.T.D.

When the star image moves in the direction of the arrow head, the light is first projected fully on the side $A B$ of the knife and is received by the phototube $P_{1}$; then the image comes near the edge $A$, and light is divided into two by the side planes $A B$ and $A C$ and received by both of the phototubes $P_{1}$ and $P_{2}$. The difference of the outputs of $P_{1}$ and $P_{2}$ takes the form shown in Figure 2 (b). In practice, the image motion is cancelled by the revolution of a plane parallel glass plate inserted in front of the knife. The phototubes take a roll to detect the small error signal of tracking revolution of the glass plate. The rotation of the plate, which corresponds to the diurnal motion of a star, is produced by combining synchronous motors in the quinary system. The guiding telescope consists of the objective $L_{1}$, the prism $P$ of which the silvered back side is used as the reflecting surface for the main telescope and an E.T.D. which 


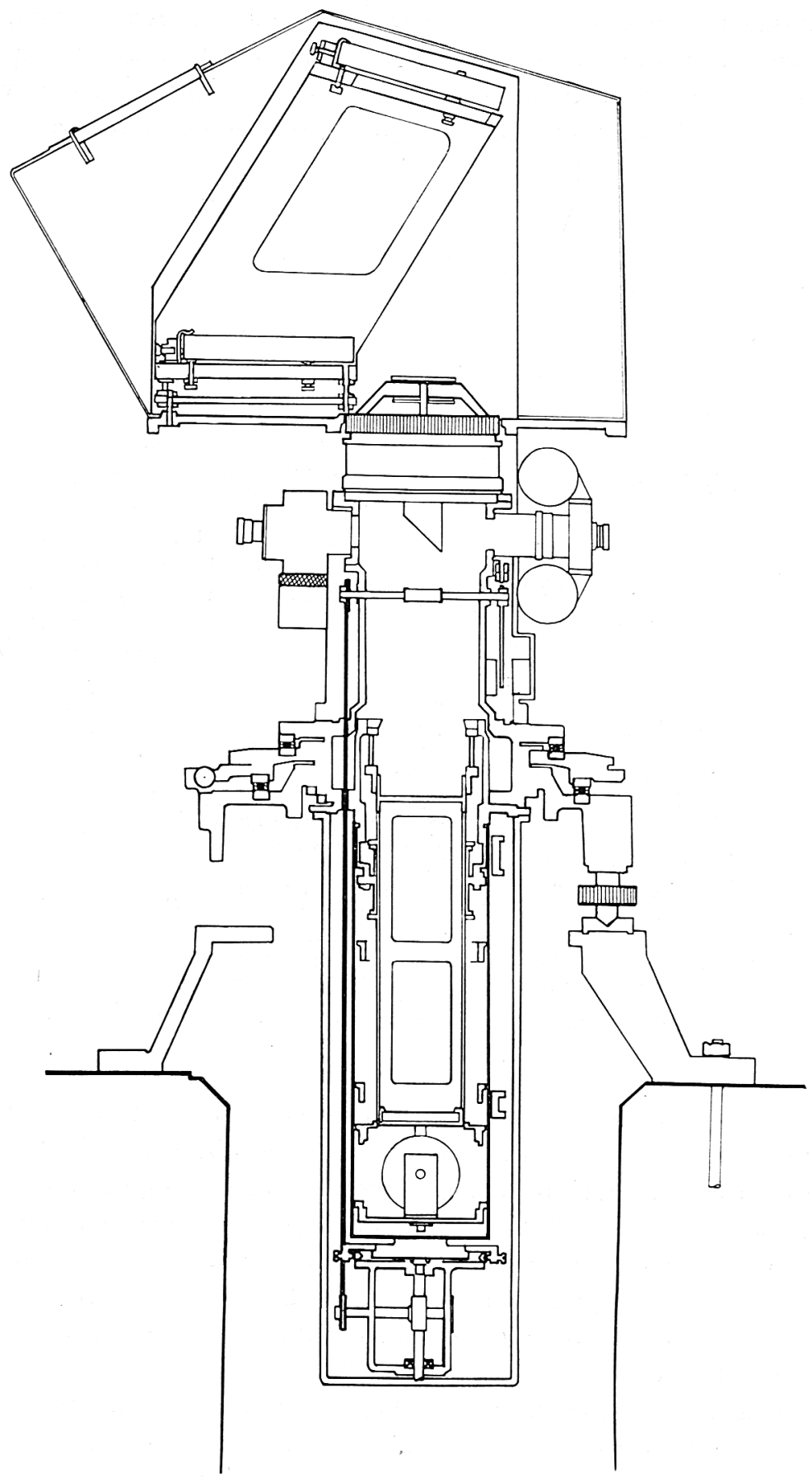

Fig. 3. The structure of the main optical part of the astrolabe. 
detects the azimuth deviation of a star image. The guiding telescope is employed to guide the star image in the azimuth direction. Figures 3 and 4 show the main optical part and the block diagram of the automatic control system of the astrolabe respectively.

Considering that the operations are to guide a star by adjusting the azimuth and to

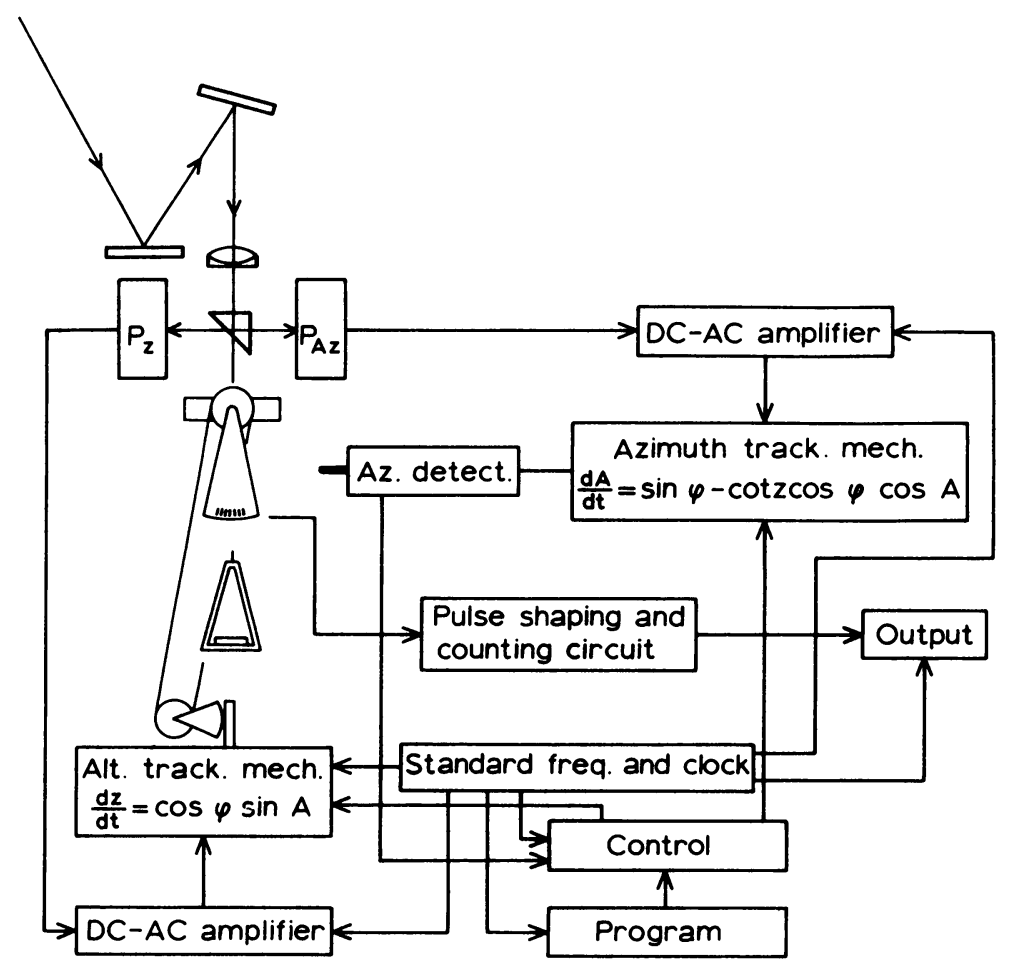

Fig. 4. Block diagram of the automatic control system of the astrolabe.

turn the telescope through $180^{\circ}$ during the passage of a star and that the period for reposing the image on the knife-edge should be made considerably long in order to integrate the effect of scintillation, the instrument should be equipped with the following mechanisms. (1) Azimuth setting mechanism for respective stars observed. (2) Mechanism which revolves the telescope by $180^{\circ}$ during the passage of a star near the mean altitude. (3) Tracking mechanism in azimuth direction. (4) Tracking mechanism in altitude direction. (5) Read-out mechanism of the times of passages of a star on the respective altitude circles.

The instrument is set on the position of the programmed azimuth by means of the worm gear driven by a pair of high and low speed motors. Tracking of a star in the azimuth direction is done by the revolution of this gear, of which the velocity is given by combining the rotation of the motor mechanism of variable velocity and the motion of the servo-system which is actuated by a correctional photoelectric signal of 
a star image detected by the E.T.D. of the guiding telescope (Figures 4 and 5(b)). Tracking in the altitude direction directly affects the observational accuracy. So the revolution of the plane parallel plate is very precisely controlled to keep the image of a star on the edge of a knife. The velocity of revolution is given in the same way as for the azimuth direction as shown in Figure 5(a). The components of diurnal angular velocity of a star are written as:

$$
\frac{\mathrm{d} A}{\mathrm{~d} t}=\sin \varphi-\cot z \cdot \cos \varphi \cdot \cos A \quad \text { Azimuth direction }
$$
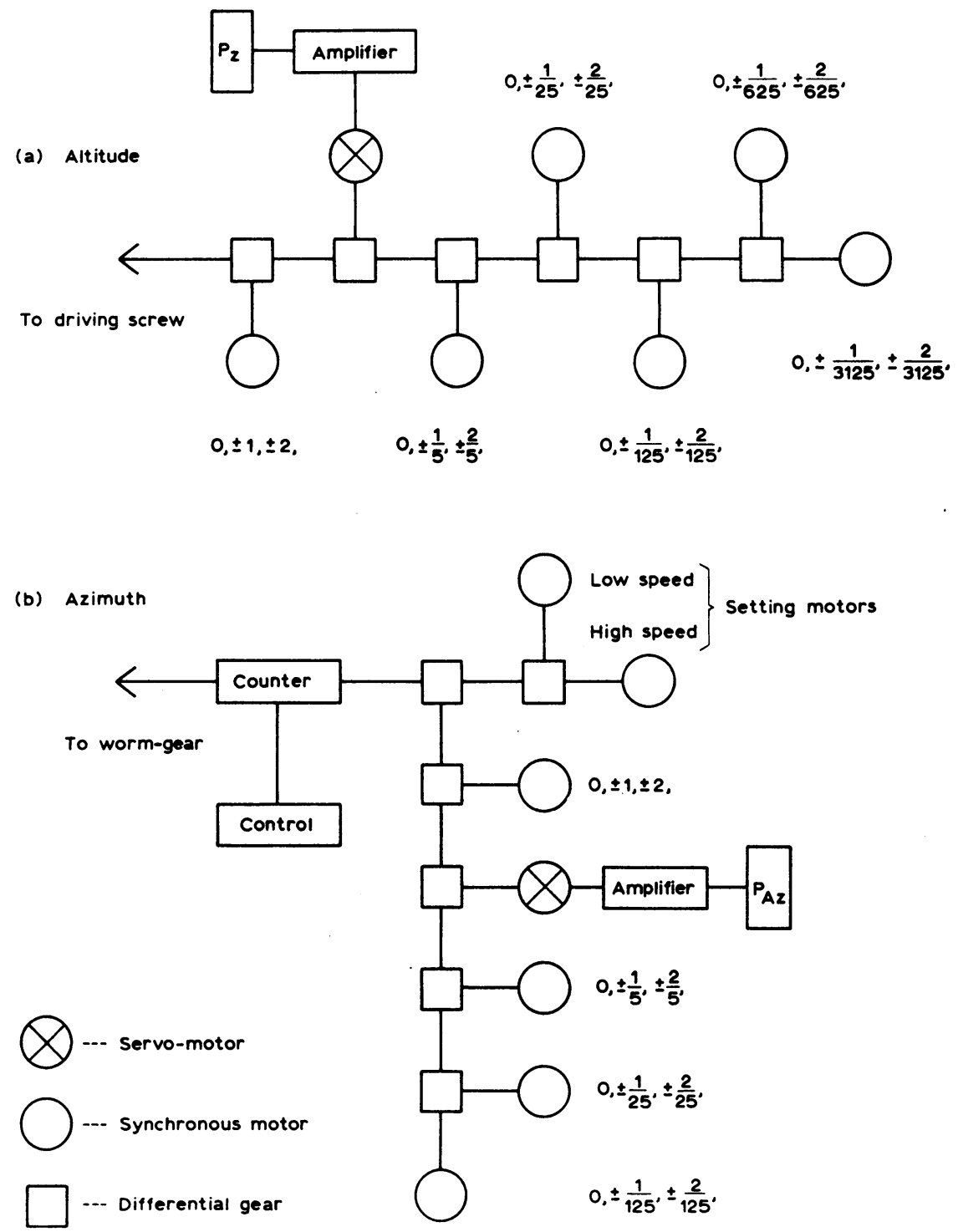

Fig. 5. Motor mechanisms for tracking star image. 


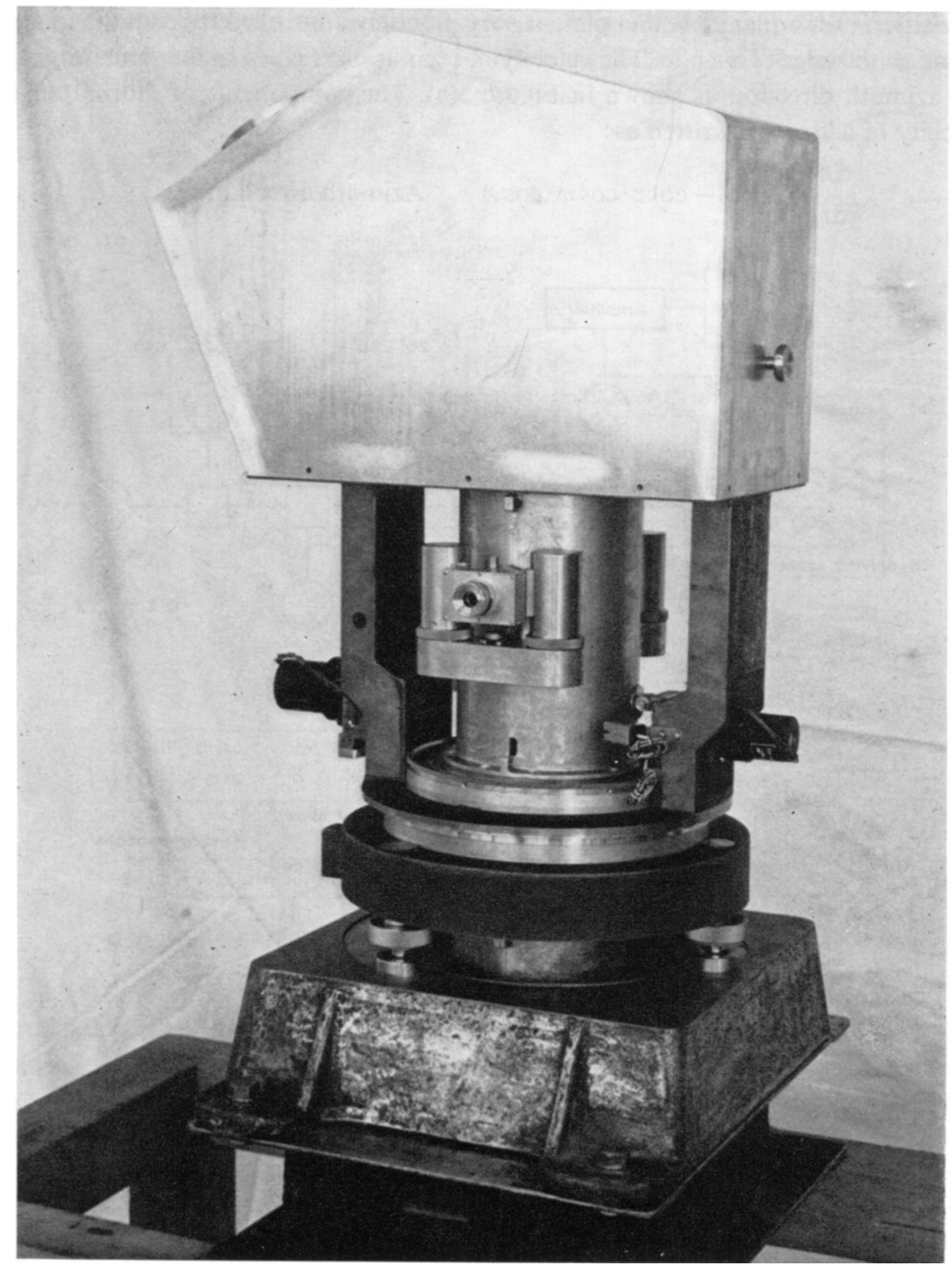

Fig. 6. The main optical part of the astrolabe (under construction). 


$$
\frac{\mathrm{d} z}{\mathrm{~d} t}=\cos \varphi \cdot \sin A
$$

Altitude direction

These component velocities are produced by the motor mechanisms of variable velocity as shown in Figure 5. Each consists of a set of synchronous motors combined with differential gears in quinary system. The synchronous motors are arranged so that their revolutions are diminished succesively to one fifth of the preceding one. Each motor has 5 modes of revolution, that is to say, 0 (repose), \pm 1 (direct and reverse motion of the first velocity), and \pm 2 (direct and reverse of the twice of the former). With this mechanism tracking accuracy of $0.1 \mathrm{~ms}$ per $1 \mathrm{~s}$ may be obtained in altitude direction. When the image attains to the edge of the knife, the converter gives such precise tracking revolution that it is able to make the speed of response of the servosystem fairly slow. If it is taken as $1 \mathrm{cps}$, the $\mathrm{S} / \mathrm{N}$ ratio will be improved more than 5 times compared with the field type instrument, which uses a direct recording oscillograph of about $30 \mathrm{cps}$. The effect of scintillation faster than $2 \mathrm{cps}$ will be almost eliminated.

The informations on the altitudes where an observed star passes is contained in the angular position of the plane parallel plate. These positions are indicated by the graduations on the fan-shaped glass plate, the center of which is fixed to the rotational axis of the plane parallel plate. The times when the graduations pass in front of a slit are read with an electro-optical detector and punched out on a paper or magnetic tape.

Figure 6 shows the appearance of the main optical part of the astrolabe which is now under construction.

\section{References}

Tsubokawa, I.: 1954a, J. Geod. Soc. Japan 1, 20.

Tsubokawa, I.: 1954b, J. Geod. Soc. Japan 1, 54.

Tsubokawa, I. and Seto, T.: 1957, J. Geod. Soc. Japan 4, 6.

Tsubokawa, I. and Dambara, T.: 1957, J. Geod. Soc. Japan 4, 15.

\section{DISCUSSION}

B. Guinot: We are also working on the development of a photo electric astrolable. We have found difficulties related to the horizontal component of the motion of the images. This component requires a very precise levelling (with accuracy of a few seconds of arc) of a compensating device. How do you solve this problem?

I. Tsubokawa: As reported before, the telescope is reversed through $180^{\circ}$ around the collimation axis before the star image reaches the central line. Owing to this procedure, requisite stability of the mechanical and optical parts of the telescope should be maintained only during the passage of a single star (less than a few minutes).

The effect of erroneous setting of the compensating mirror is eliminated in the following way. If the mirror surface is inclined by $\varepsilon$ to the horizontal plane, the reflected ray will pass through $f \varepsilon$ away from the nodal point $K_{2}$, where $f$ is the focal length of the objective (Figure 3A(a)). However, because the separation is perfectly symmetrical to $K_{2}$ in the direct and reversed positions of the telescope, the mean of observations at these two positions is independent of the separation except curvature corrections.

The effect of small inclination of the knife edge line of the detector to the horizontal line is also minimized when almost the same point on the edge line is used in the direct and reversed positions 


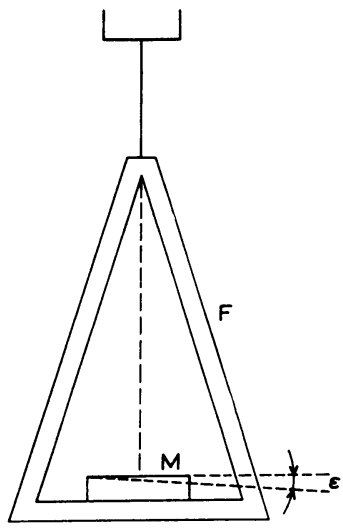

(a)
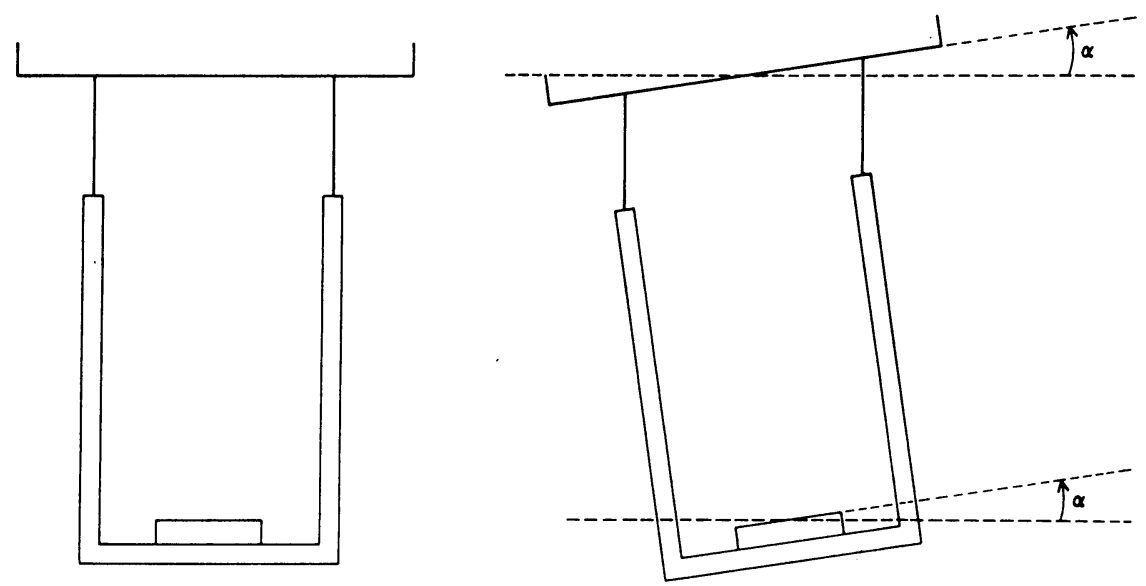

(b)

Fig. 3A.

by guiding the star image in horizontal direction. The compensating mirror is suspended pendulously by two sets of intersecting strings and the line passing through these two points of intersection is the rotational axis of the pendulum, which is parallel to the altitude circle. However, in the vertical plane containing the above mentioned axis, the pendulum is in effect suspended by two parallel strings as shown in Figure $3 \mathrm{~A}(\mathrm{~b})$. In such a configuration, the pendulum will incline through the same angle as the frame and hence also the telescope, and the inclination in this plane is not compensated. Accordingly, the angular displacements of the images of the main and the guiding telescopes are the same in horizontal direction, and no guiding error is introduced. For this reason, small inclination of the edge line of the detector of the main telescope to the horizon has negligible effect.

Imperfect parallelity between the intersection line of the mirror $M_{1}$ and $M_{2}$ in Figure 1 (deflecting mirror system) and the axis of the compensator, especially its horizontal component, may introduce more critical error than those mentioned above, which may be the error pointed out by Dr Guinot, I think. Let us suppose the parallelity error be $\alpha$ and the vertical axis error of the instrument be $i$, then the maximum observational error will be approximately $\alpha i$. If $\alpha \lesssim 1^{\prime}, i \lesssim 10^{\prime \prime}$, the error will be less than $3 \times 10^{-3}$ second of arc. Reversing error also cause the same effect. The instrument has the adjusting mechanisms which make these errors less than one minute of arc.

$S$. Débarbat: In your paper you wrote that your astrolabe "has an accuracy comparable to that of the 
Danjon astrolabe". Did you make your comparisons in the same place for instance at the Mizusawa observatory close to the Danjon's astrolabe? We are very interested in the comparison between different instruments. As Mizusawa has a lot of instruments giving results for the BIH and for the IPMS (FZT, VZT, PZT, Astrolabe (Danjon)), I thought that, perhaps, you have made your comparisons in the Mizusawa observatory.

I. Tsubokawa: Observations with the field type instruments (effective aperture is about $50 \mathrm{~mm}$, $f=800 \mathrm{~mm}$ ) show the inner accuracy of about $7^{\mathrm{ms}}$ in longitude and about 0.1 in latitude, as the standard deviations of a single group.

Preliminary parallel observations with Danjon and Tsubokawa astrolabes were made at Mizusawa (I.L.O.) observatory in 1965 and 1966 . Observed stars do not perfectly coincide with each other because of the bad observing conditions.

The following table shows the values of $\varphi$ and $\lambda$, determined in these observations, reduced to the site of the Danjon Astrolabe.

\begin{tabular}{|c|c|c|c|c|c|c|}
\hline \multicolumn{3}{|c|}{ Danjon Astrolabe } & \multicolumn{2}{|c|}{ Tsubokawa Astrolabe } & \multirow{2}{*}{$\frac{\text { Danjon }}{\text { Longitude }}$} & \multirow{2}{*}{$\begin{array}{l}\text { Tsubokawa } \\
\text { Longitude }\end{array}$} \\
\hline Date & $\begin{array}{l}\text { No. } \\
\text { of stars }\end{array}$ & Latitude & $\begin{array}{l}\text { No. } \\
\text { of stars }\end{array}$ & Latitude & & \\
\hline \multicolumn{7}{|c|}{1965} \\
\hline $\begin{array}{l}\text { Nov. } 13 \\
\text { Nov. } 16 \\
\text { Nov. } 17\end{array}$ & $\begin{array}{l}24 \\
22 \\
14 \\
14 \\
18\end{array}$ & $\begin{array}{r}39^{\circ} 08^{\prime} 3^{\prime \prime} .59 \\
3^{\prime \prime} .73 \\
3^{\prime \prime} .05 \\
3^{\prime \prime} .46 \\
3^{\prime \prime} .60\end{array}$ & $\begin{array}{l}26 \\
22 \\
18 \\
21 \\
20\end{array}$ & $\begin{array}{r}39^{\circ} 08^{\prime} 3^{\prime \prime} .48 \\
3^{\prime \prime} .33 \\
3^{\prime \prime} .50 \\
3^{\prime \prime} .31 \\
3 . " 57\end{array}$ & $\begin{array}{r}9^{\mathrm{h}} 24^{\mathrm{m}} 31^{\mathrm{s}} .594 \\
.586 \\
.574 \\
.632 \\
.624\end{array}$ & $\begin{array}{r}9^{\mathrm{h}} 24^{\mathrm{m}} 31^{\mathrm{s}} .592 \\
.560 \\
.594 \\
.590 \\
.575\end{array}$ \\
\hline Mean & & $3^{\prime \prime} .49 \pm 0^{\text {p.e. }} .09$ & & $3^{\prime \prime} .44 \pm 0^{\prime \prime} .04$ & $.602 \pm 0.8005$ & $.582 \pm 0 .{ }^{5004}$ \\
\hline \multicolumn{7}{|c|}{1966} \\
\hline $\begin{array}{l}\text { Apr. } 30 \\
\text { May } 4 \\
\text { May } 5\end{array}$ & $\begin{array}{l}12 \\
14 \\
21 \\
16 \\
18 \\
16\end{array}$ & $\begin{array}{r}39^{\circ} 08^{\prime} 3^{\prime \prime} .00 \\
3^{\prime \prime} .26 \\
3^{\prime \prime} .78 \\
3^{\prime \prime} .19 \\
3^{\prime \prime} .44 \\
3^{\prime \prime} .69\end{array}$ & $\begin{array}{l}\overline{18} \\
24 \\
18 \\
22 \\
25\end{array}$ & $\begin{array}{r}-79^{\circ} 08^{\prime \prime} 3^{\prime \prime} .70 \\
3^{\prime \prime} .69 \\
3^{\prime \prime} .38 \\
3^{\prime \prime} .50 \\
3^{\prime \prime} .89\end{array}$ & $\begin{array}{r}9^{\mathrm{h}} 24^{\mathrm{m}} 31^{\mathrm{s}} .516 \\
.526 \\
.497 \\
.526 \\
.521 \\
.542\end{array}$ & $\begin{array}{r}- \\
9^{\mathrm{h}} 24^{\mathrm{m}} 31^{\mathrm{s}} .564 \\
.533 \\
.557 \\
.533 \\
.525\end{array}$ \\
\hline Mean & & $\begin{array}{c}\text { p.e. } \\
3^{\prime \prime} .39 \pm 0^{\prime \prime} .08\end{array}$ & & $3^{\prime \prime} .63 \pm 0^{\prime \prime} .06$ & $.526 \pm 0.8003$ & $.542 \pm 0.8005$ \\
\hline
\end{tabular}

